\title{
SOX2 contributes to melanoma cell invasion
}

\author{
Sasha D Girouard ${ }^{\text {, Alvaro C Laga }}{ }^{1}$, Martin C Mihm², Richard A Scolyer ${ }^{3}$, John F Thompson ${ }^{4}$, Qian Zhan ${ }^{1}$, \\ Hans R Widlund ${ }^{2}$, Chung-Wei Lee ${ }^{1 *}$ and George F Murphy ${ }^{1 *}$
}

The mechanisms of melanoma invasion are poorly understood despite extensive inquiry. SRY (sex determining region Y)box 2 (SOX2) is an embryonic stem cell transcription factor that has recently been discovered to be expressed in human melanoma where it is associated with dermal invasion and primary tumor thickness. To assess the potential role of SOX2 expression in melanoma invasion, we examined patient melanomas and humanized melanoma xenografts, and noted preferential SOX2 expression in cells that interfaced and infiltrated dermal stroma. Experimental knockdown (KD) of SOX2 mRNA and protein in A2058 melanoma cells with high constitutive SOX2 expression resulted in 4.5-fold decreased invasiveness in vitro compared with controls $(P<0.0001)$. Conversely, when $\mathrm{G} 361$ cells that normally express low SOX2 were transduced to overexpress SOX2 mRNA and protein, a 3.8-fold increase in invasiveness was observed $(P=0.0004)$. Among 84 invasion-related genes, RT-PCR screening revealed that SOX2 KD resulted in striking decrease in matrix metalloproteinase-3 (MMP-3), an endopeptidase associated with cleavage of the extracellular matrix. Quantitatively, SOX2 KD diminished MMP-3 mRNA by 87.8\%. MMP-3 KD in SOX2-expressing A2058 cells served to inhibit invasion, although to a lesser degree than SOX2 KD. Finally, immunostaining of patient and xenograft melanomas revealed coordinate SOX2 and MMP-3 expression in regions of stromal infiltration. These data implicate SOX2 expression in melanoma invasion, and suggest a role for MMP-3 as one potential mediator of this process.

Laboratory Investigation (2012) 92, 362-370; doi:10.1038/labinvest.2011.188; published online 19 December 2011

KEYWORDS: invasion; melanoma; matrix metalloproteinase-3; SOX2

The dominant measure of melanoma virulence conventionally has been related to extent of dermal invasion, as assessed either by Clark levels or by the Breslow measurement of tumor thickness. However, identification of genomic factors that determine and regulate invasive potential has remained elusive despite intensive and extensive study. Because cancer cell virulence may involve pathological expression of genes that normally govern migration of progenitor cells during embryogenesis, evaluation of such mechanisms could provide insight into how melanoma evolves from an initially curable superficial lesion to a more deeply invasive form associated with metastasis and mortality.

SOX2 is a key regulatory gene located on chromosome 3q26.33 that encodes a transcription factor important for embryonic stem cell pluripotency and for maintenance of physiologically migratory neural progenitor cells. ${ }^{1-3}$ Since its initial characterization in embryogenesis and development, SOX2 expression has been implicated in poorly differentiated malignancies affecting a variety of organs. ${ }^{4-10}$ Notably, SOX2 is amplified in lung, esophageal, and oral squamous cell carcinomas, in which it may in part function as a lineagesurvival oncogene. ${ }^{11-13}$

SOX2 was recently discovered to be preferentially expressed in human melanoma, where it was found to be present in up to $67 \%$ of primary melanomas and $80 \%$ of metastatic melanomas, compared with $14 \%$ of nevi. ${ }^{14,15}$ Moreover, in preliminary analyses SOX2 immunopositivity correlated with dermal invasion, as assessed by increased tumor thickness, a key marker of prognosis. ${ }^{14}$ In support, a recent bioinformatics analysis of the expression of stem cell markers in 40 different human cancers revealed that the 3-year median survival for patients with SOX2-expressing metastatic melanoma was 145 days less than that of patients with SOX2negative metastatic tumors, ${ }^{16}$ also suggesting that expression of the stem cell-associated SOX2 transcription factor relates to melanoma virulence. We have preliminarily observed a

${ }^{1}$ Program in Dermatopathology, Department of Pathology, Brigham and Women's Hospital, Harvard Medical School, Boston, MA, USA; ${ }^{2}$ Department of Dermatology, Brigham and Women's Hospital, Harvard Medical School, Boston, MA, USA; ${ }^{3}$ Tissue Pathology and Diagnostic Oncology, Royal Prince Alfred Hospital; Discipline of Pathology, The University of Sydney; and The Poche Centre, Melanoma Institute Australia, Sydney, NSW, Australia; ${ }^{4}$ Melanoma Institute Australia and Discipline of Surgery, The University of Sydney, Sydney, Australia

Correspondence: Dr C-W Lee or Professor GF Murphy, Brigham and Women's Hospital, Program in Dermatopathology, 221 Longwood Avenue (EBRC Suite 401), Boston, MA 02115, USA.

E-mail: clee29@partners.org (C-WL) or gmurphy@rics.bwh.harvard.edu (GFM)

*These are co-senior authors.

Received 21 September 2011; revised 28 October 2011; accepted 2 November 2011 
tendency for SOX2 expression to favor more invasive melanoma phenotypes, and recent evidence suggests that the more invasive sub-populations within cancers might be associated with or even induce stem cell-like properties. ${ }^{17-19}$

Despite such preliminary associations with tumor depth and virulence, the precise function of SOX2 in melanoma remains unclear. Recent data in lung squamous cell carcinomas indicates that SOX2 functions as an oncogene that activates embryonic stem cell phenotypes, in doing so it provides clues to the deregulated downstream genes involved in the malignant phenotype. ${ }^{11}$ Given the relationship of SOX2 to normally migratory neural crest progenitors and its apparent preferential association with more infiltrative, matrix-associated melanoma sub-populations, we hypothesized that SOX2 expression may relate to melanoma invasion. This study was designed to preliminarily investigate this possibility.

\section{MATERIALS AND METHODS Human Samples}

Paraffin-embedded sections of five nodular and five desmoplastic human melanomas were obtained from the Melanoma Institute Australia Biospecimen Bank (Sydney, Australia), and four superficial spreading melanomas were obtained from the Department of Pathology, Brigham and Women's Hospital. All patient tissue was obtained according to an approved Institutional Review Board protocol. A human melanoma tissue microarray (TMA) containing 37 evaluable cores annotated according to primary $v s$ metastatic melanoma origin and with survival outcomes was evaluated (Imgenex, San Diego, CA, USA).

\section{Cell Lines and Cell Growth In Vitro}

Human melanoma cell lines A2058, A375, and G361 were obtained from American Type Culture Collection (Manassas, VA, USA) and were grown in Dulbecco's modified Eagle's medium (DMEM; Sigma-Aldrich, St Louis, MO, USA), supplemented with $10 \%$ heat inactivated fetal bovine serum (FBS; Hyclone laboratories, Logan, UT, USA), and $200 \mathrm{mM} / \mathrm{l}$ L-glutamine, $100 \mathrm{IU} / \mathrm{ml}$ penicillin and $100 \mu \mathrm{g} / \mathrm{ml}$ streptomycin, and maintained at $37^{\circ} \mathrm{C}$ in a humidified atmosphere containing $5 \% \mathrm{CO}_{2}$. To measure conventional monolayer cell growth, subconfluent cultures were trypsinized and seeded in 35 -mm wells at $2 \times 10^{4}$ cells per well. Cells were re-fed twice weekly. At given intervals, cells in quadruplicate wells were collected and counted with a hematocytometer. For assessment of proliferation, cells were seeded on 96-well tissue culture plates at the density of 2000 cells/well overnight. Viable cells were quantified by the metabolic cell counting dye (Kit 8, Dojindo, Rockville, MD, USA) using the Vector3 plate reader (Perkin-Elmer, Waltham, MA, USA) at $560 \mathrm{~nm}$.

\section{Manipulation of Gene Levels in Melanoma Cells}

Lentivirus-based shRNA method was used for gene knockdown (KD) of Sox2 and matrix metalloproteinase-3
(MMP3). pLKO-pruo-shSOX2 or pLKO-pruo-shMMP3 lentiviral vector (Sigma-Aldrich) was co-transfected with packaging vectors psPAX2, pMD2.VSV-G in HEK293T cells using FuGene6 (Roche Applied Science, Indianapolis, IN, USA). pLKO-puro-scramble was used as control. To overexpress SOX2, human SOX2 cDNA was excised from plasmid pSIN-EF2-SOX2 (Addgene plasmid \#16577) using BstB1 and blunted followed by EcoRI digestion, and cloned into retroviral pBABE-puro backbone digested by EcoRI and SalI (blunted). pBabe-puro-Sox2 was co-transfected with packaging vectors pCMV-VSV-G and pUMVC3 in HEK293T cells using Fugene6. Viral supernatants were collected, filtered through $0.45 \mu \mathrm{m}$ sterile filter, and used together with polybrene $(8 \mathrm{mg} / \mathrm{ml})$ to transduce target cells. Transduced cells were selected with puromycin $(1 \mathrm{mg} / \mathrm{ml})$ starting $48 \mathrm{~h}$ post infection and maintained in the presence of the antibiotic. Manipulation of SOX2 and MMP3 levels was verified by quantitative RT-PCR and western blotting, and low-passage cell cultures (passages $\leq 5$ ) were used throughout experiments.

\section{Human Melanoma Xenotransplantation}

Melanoma cells from A2058-SOX2-KD $(n=5)$ and A2058scramble (control; $n=5$ ) cell lines were subcutaneously injected into severe combined immunodeficient mice, as described previously. ${ }^{15}$ Human to chimeric mouse/human skin melanoma xenotransplantation of A2058 melanoma cells $(n=5)$ was also evaluated, as described previously. ${ }^{15}$ All mice were maintained under defined conditions according to the Harvard University Animal Use Committee guidelines, and approved IRB protocols.

\section{Quantitative RT-PCR Assays}

Total RNA from the indicated human melanoma cell lines was extracted using the RNAeasy Mini Kit (Qiagen, Valencia, CA, USA) and cDNA generated using the SuperScript III First-strand Synthesis System for RT-PCR (Invitrogen, Carlsbad, CA, USA). Specifcally, SOX2 and MMP-3 expression was surveyed using the SOX2 (Cat. No. Hs01053049-s1) and MMP-3 (Cat. No. Hs00968305_ml) assays for real-time RT-PCR (Applied Biosystems, Foster City, CA). Quantitative RT-PCR was performed on a 7300 Real-Time PCR System (Applied Biosystems) in a $25-\mu \mathrm{l}$ reaction mix containing $1 \mu \mathrm{l}$ cDNA, $1 \times$ TaqMan Universal PCR Master Mix, and $1 \times \operatorname{SOX} 2$ or MMP- 3 or $\beta$-actin assay. The $\beta$-actin reference gene was used for normalization and data were analyzed with the $\Delta \Delta C_{\mathrm{t}}$ method. ${ }^{20}$ Comparison of the relative mRNA expression of 84 genes related to epithelial-mesenchymal transition (EMT; a phenomenon often linked to stromal invasion) between the control and SOX2-KD A2058 cell lines was made using Human EMT RT ${ }^{2}$ Profiler PCR Array (Cat. No. PAHS-090, SA Biosciences, Frederick, MD, USA).

\section{Western Blotting}

Subconfluent cultures were washed with PBS and extracted in $1 \times$ lysis buffer (Cell Signaling, Danvers, MA, USA) 
containing $1 \mathrm{mM}$ phenylmethylsulfonyl fluoride. Cell lysates were quantified using a bicinchoninic acid protein assay kit (Pierce, Rockford, IL, USA). An equal amount $(75 \mu \mathrm{g})$ of total protein from each sample was subjected to electrophoresis on NuPAGE 4-12\% Bis-Tris gels (Invitrogen), transblotted onto nitrocellulose membranes (Pierce), and probed with primary rabbit anti-SOX2 antibody (Cell Signaling), or rabbit anti-MMP-3 (Abcam, Cambridge, MA, USA) followed by a peroxidase-conjugated secondary antibody (Vector Laboratories, Burlingame, CA, USA). Subsequent re-probing using anti- $\beta$-actin antibody (Abcam) was performed as internal loading control. Immunoreactive bands were detected using SuperSignal West Pico Chemiluminescent Substrate (Pierce), captured by a Syngene Chem Genius Bio Image System (Syngene, Frederrick, MD, USA) and quantified by densitometry.

\section{Immunohistochemistry and Immunoflourescence}

Goat anti-SOX2 antibody (Neuromics, Edina, MN, USA) and rabbit anti-MMP-3 antibody (Abcam) were used for staining $5-\mu \mathrm{m}$ paraffin sections of patient and xenograft melanomas. Immunohistochemical staining was performed as previously described. ${ }^{14}$ For double-label immunohistochemical staining, sections were incubated with SOX2 and MMP-3 antibodies at $4^{\circ} \mathrm{C}$ overnight and then incubated with peroxidaseconjugated horse anti-goat IgG (Vector) and alkaline phosphatase-conjugated donkey anti-rabbit IgG secondary antibodies (Abcam) at room temperature for $30 \mathrm{~min}$. SOX2 and MMP-3 staining were detected with NovaRED Peroxidase Substrate Kit and Blue Alkaline Phosphatase Substrate Kit (Vector).

For immunofluorescence double labeling of xenograft melanomas, $5-\mu \mathrm{m}$ paraffin sections were incubated with Sox 2 and MMP-3 antibodies at $4{ }^{\circ} \mathrm{C}$ overnight and then incubated with Alexa Fluor 594-conjugated anti-goat IgG and Alexa Fluor 488-conjugated anti-rabbit IgG at room temperature for $1 \mathrm{~h}$. The sections were coverslipped with ProLong Gold anti-fade with DAPI (Invitrogen). Sections were analyzed with a BX51/BX52 microscope (Olympus America, Melville, NY, USA), and images were captured using the CytoVision 3.6 software (Applied Imaging, San Jose, CA, USA).

\section{Invasion Assays}

Subconfluent cultures were collected by careful trypsin digestion and resuspended in $0.1 \%$ BSA-DMEM culture medium. Cells $\left(2.5 \times 10^{4}\right.$ cells for A2058-derived cell lines and $5.0 \times 10^{4}$ cells for A375- and G361-derived cell lines) were seeded into the upper compartments of either BD BioCoat Growth Factor Reduced Matrigel Invasion Chambers or BD BioCoat Control Inserts (BD Biosciences, Bedford, MA, USA), and DMEM supplemented with 10\% FBS was added to the lower compartment according to the manufacturer's instructions. The invasion chambers and control inserts were incubated for $8-24 \mathrm{~h}$ at $37^{\circ} \mathrm{C}$ in a humidified atmosphere containing $5 \% \mathrm{CO}_{2}$. After incubation, the non-invading cells were removed from the upper surface of the membrane by gentle scrubbing, and the cells on the lower surface of the membrane were fixed in $10 \%$ formalin and stained with hematoxylin Grill\#1 and 0.1\% ammonium hydroxide. Cell counting was facilitated by photographing the membrane through the microscope at the area of highest cell density, and triplicate membranes were counted under $\times 200$ magnification per experiment. The results were averaged, and the mean of two experiments is shown for each cell line. The percent invasion was calculated by dividing the mean number of cells that invaded through the Matrigel insert membrane by the mean number of cells that migrated through the control insert.

\section{Statistical Analyses}

Data presented are means \pm s.d. Statistically significant differences were assessed by Student's $t$-test and the $\alpha$ level was set at $<0.05$ in all cases.

\section{RESULTS \\ SOX2 is Associated with Human Melanoma Cell Invasion In Situ}

We initially examined the distribution of SOX2-expressing cells in situ in patient melanomas $(n=10)$ and in a model whereby experimental human melanomas develop after injection of melanoma cell lines into the dermis of human skin xenografts $(n=5)$ established on the backs of immunocompromised mice, a system that permits assessment of human melanoma cells in the context of an intact human dermal microenvironment. ${ }^{21}$ Cells expressing nuclear immunoreactivity for SOX2 in patient melanomas tended to show spindled ( $v s$ epithelioid) contours and to be more concentrated at tumor-stromal interfaces (Figures 1a-c). In xenografts, SOX2-positive cells were consistently concentrated at the perimeter of tumor nodules where they infiltrated among bundles of the human dermal collagen (Figures 1d and e). Faint cytoplasmic background staining was noted both with anti-SOX2 antibody and in negative controls, and thus was concluded to be nonspecific.

\section{SOX2 Depletion Inhibits Functional Human Melanoma Cell Invasion}

We next examined how expression of SOX2 related to melanoma cell invasion using a conventional in vitro Matrigel assay. In initial experiments, a cell line was selected that expressed relatively high levels of SOX2 as determined by real-time RT-PCR and western blotting (A2058). To examine the specific effects of SOX2 on invasion, we utilized a lentiviral/shRNA approach to silencing SOX2 gene expression in the A2058 cells. Real-time RT-PCR and western blotting demonstrated decreased SOX2 expression in the A2058 SOX2-KD line, with $91.4 \%$ efficiency by densitometry (Figures 2a and b). Knockdown of SOX2 in A2058 melanoma cells was associated with significant inhibition of invasion 

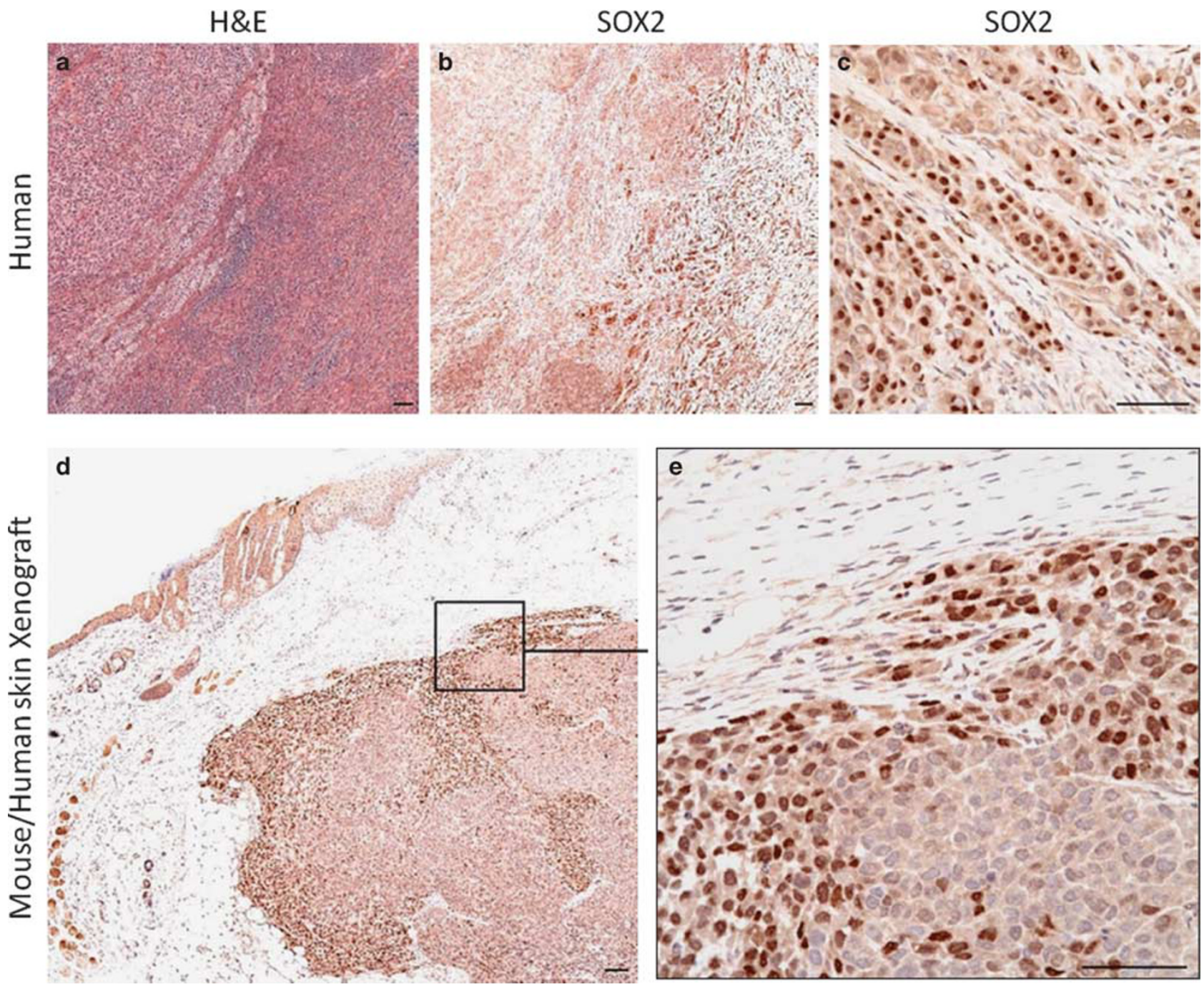

Figure 1 Example of SOX2 immunoreactivity in patient and xenograft melanomas. Haematoxylin and eosin (H\&E) staining of biphasic patient melanoma with more epithelioid region to the left of the field and more fusiform region to the right of the field (a). There is preferential nuclear immunoreactivity for SOX2 in the fusiform region to the right of the field $(\mathbf{b}, \mathbf{c})$. Humanized melanoma xenograft shows accentuation of SOX2 immunoreactivity along tumor perimeter and in association with zones of stromal invasion (d, e; $\mathbf{d}$ inset: H\&E showing melanoma nodule growing within dermis of human skin xenograft flanked on both sides by skin of murine recipient). (a-c, e) Bar $=50 \mu \mathrm{m},(\mathbf{d}) \mathrm{bar}=100 \mu \mathrm{m}$.

in vitro by $78 \%(P<0.0001)$ relative to vector-infected controls (Figures $2 \mathrm{c}$ and $\mathrm{d}$ ). To minimize the potential influence of cell proliferation, the invasion experiments were replicated at $8 \mathrm{~h}$ time points, which also showed inhibition of invasion (94\%; $P<0.0001)$. Of note, cell doubling time in monolayer in vitro over a $24-\mathrm{h}$ period was not affected by SOX KD, as previously shown, ${ }^{14}$ and median cell cycle time for the A2058 line was calculated to be between 20 and $22 \mathrm{~h}$ for both vector and $\mathrm{KD}$ lines. To insure reproducibility of these findings in a second melanoma line that also constitutively expressed SOX2, KD of SOX2 protein in A375 melanoma cells was accomplished (75\% efficiency) and also resulted in significantly reduced invasion in vitro by $79 \%$ $(P<0.001)$ compared with vector-infected controls (data not shown).

\section{SOX2 Overexpression Enhances Functional Human Melanoma Cell Invasion}

On the basis of the SOX2-KD data, we reasoned that overexpression of SOX2 may enhance invasion within melanoma cells that normally express low levels of this factor. First, we overexpressed SOX2 in the G361 melanoma cell line that has relatively low levels of endogenous SOX2 expression by RT-PCR and western blotting $79.4 \%$ and $78.3 \%$ reduction, respectively), as compared with A2058 cells (Figures 2e and f). SOX2 overexpression was confirmed in the transduced cell line, with a 7.5-fold increase in SOX2 protein over vector-treated controls (Figures $2 \mathrm{~g}$ and $\mathrm{h}$ ). In keeping with our hypothesis, overexpression of SOX2 in G361 melanoma cells was associated with enhanced invasion in vitro by 3.8 -fold $(P=0.0004)$ relative to controls (Figures $2 \mathrm{i}$ and $\mathrm{j}$ ). 


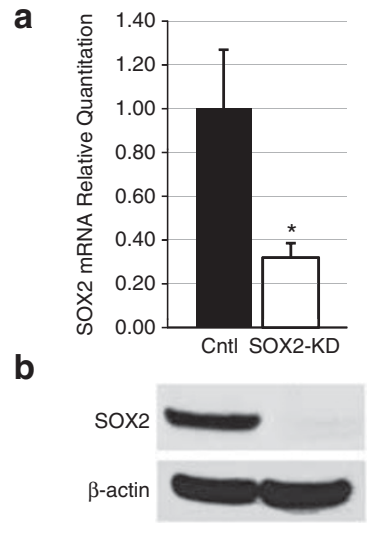

e

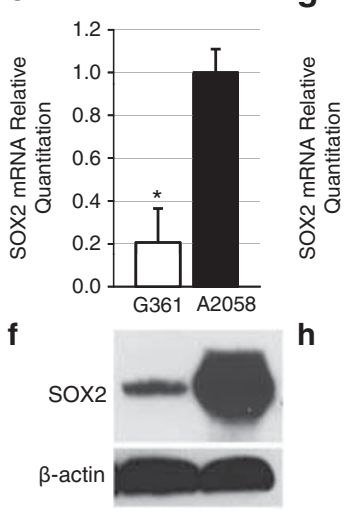

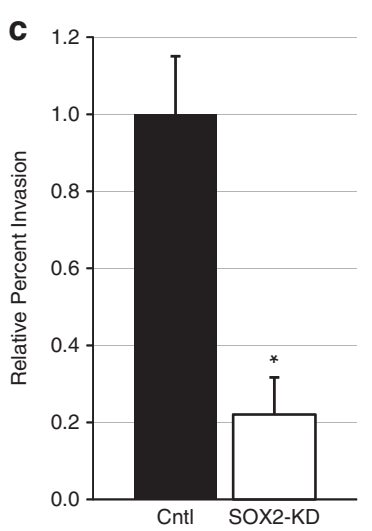

i

d

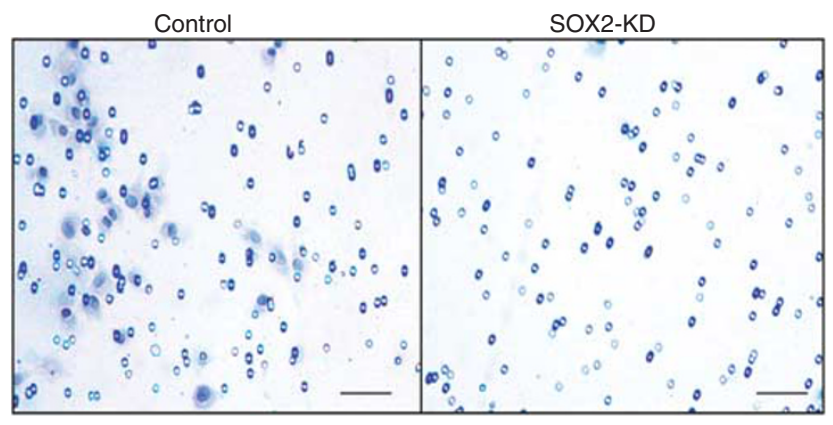

$\mathbf{j}$

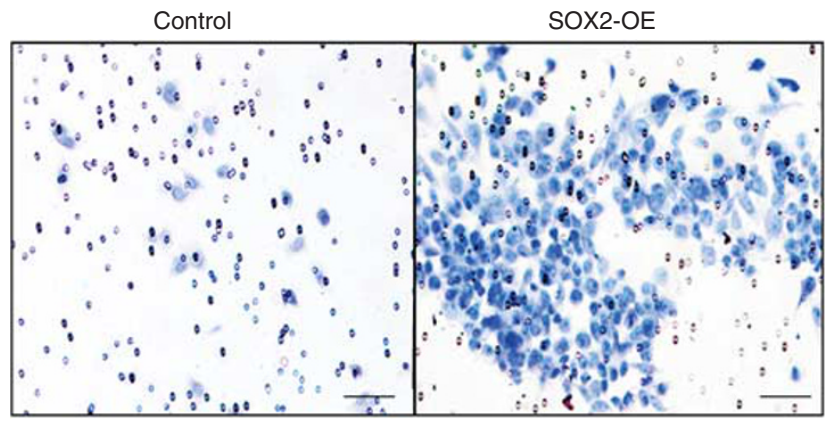

Figure 2 SOX2 expression and invasion. SOX2 knockdown (KD) of A2058 melanoma cells was confirmed via real-time RT-PCR (a) and western blotting $(\mathbf{b} ; \beta$-actin $=$ control). Percent invasion of A2058 SOX2-KD line was measured in Growth Factor Reduced Matrigel Invasion Chamber and compared with control (c, d). Endogenous SOX2 expression in the A2058 and G361 wild-type melanoma cell lines was shown by real-time RT-PCR (e) and western blotting (f). Overexpression of SOX2 in G361 cells was established by real-time RT-PCR (g) and western blotting (h), and invasion of SOX2-overexpressing cells was compared with control by invasion assay $(\mathbf{i}, \mathbf{j})$. Data are expressed as mean \pm s.d. ${ }^{\star}$ Marks significant $(P<0.05)$ differences compared with the control. (d, j) Bar $=100 \mu \mathrm{m}$.

Thus, the potential for SOX2 expression to correlate with an invasive phenotype was supported by these in vitro assays.

\section{SOX2 Expression Is Associated with MMP-3 that Affects Melanoma Invasion}

In an attempt to identify downstream effectors of SOX2associated melanoma invasion, we performed a candidate expression-based analysis of the SOX2-KD A2058 cell line using a sampling of 84 invasion-related genes. The most striking association among the examined genes was a significant decrease in MMP-3 expression in the SOX2-KD A2058 cells compared with vector-treated controls (Figure 3a). Quantitative RT-PCR demonstrated markedly decreased MMP-3 mRNA expression as compared with controls (a reduction of $87.8 \%$, Figure $3 \mathrm{~b}$ ); this was also confirmed by western blot analysis at a protein level (Figure 3c). Hence, taken together, these data establish a relationship between SOX2 expression, melanoma cell invasion, and MMP-3 expression.
We next reasoned that if MMP-3 was a factor in SOX2related melanoma cell invasion, MMP-3 KD in A2058 cells should have effects similar to that of SOX2 KD. Accordingly, MMP-3 KD cells were generated, with real-time RT-PCR and western blotting demonstrating decreased MMP-3 expression with $>95 \%$ efficiency by densitometry (Figures $3 \mathrm{~d}$ and e). MMP-3 KD was associated with inhibition of invasion in the in vitro assay by $55 \%(P<0.0001)$ relative to vector-infected controls (Figures $3 \mathrm{f}$ and $\mathrm{g}$ ).

\section{SOX2 and MMP-3 Exhibit Co-Expression within Patient and Xenograft Melanomas}

We next sought to determine whether the relationship between SOX2 and MMP-3 was replicated in melanoma tissue. Using dual-labeling immunohistochemistry, the patterns of SOX2 and MMP-3 expression were assessed in vivo by qualitative analysis of 14 patient melanomas. Five were desmoplastic melanomas with marked degrees of stromal infiltration, and nine were either nodular $(n=5)$ or superficial spreading $(n=4)$ melanomas dominated by relatively 


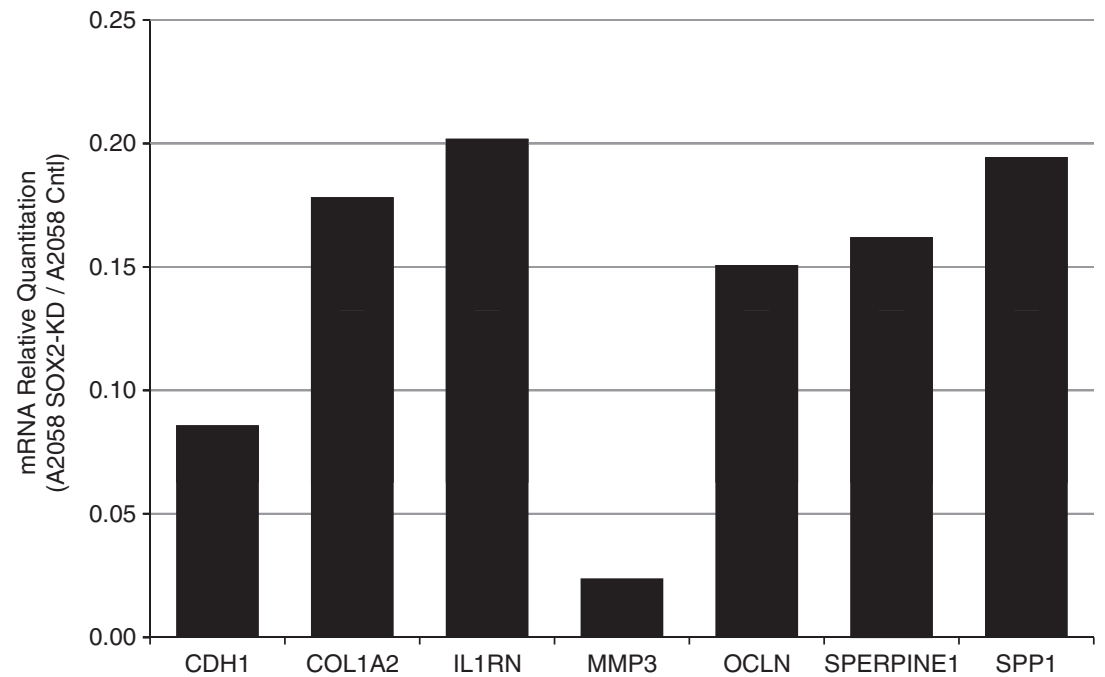

b

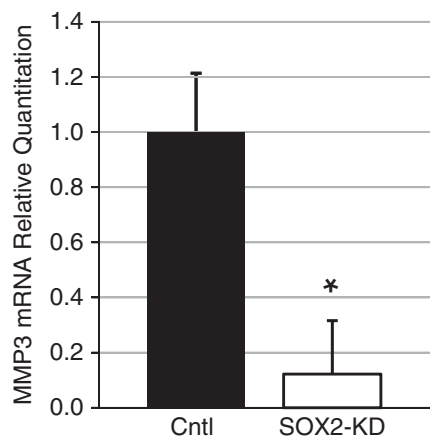

C

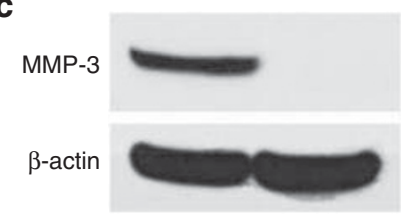

d

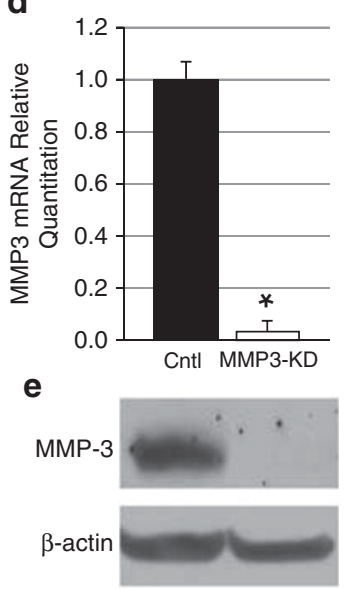

f
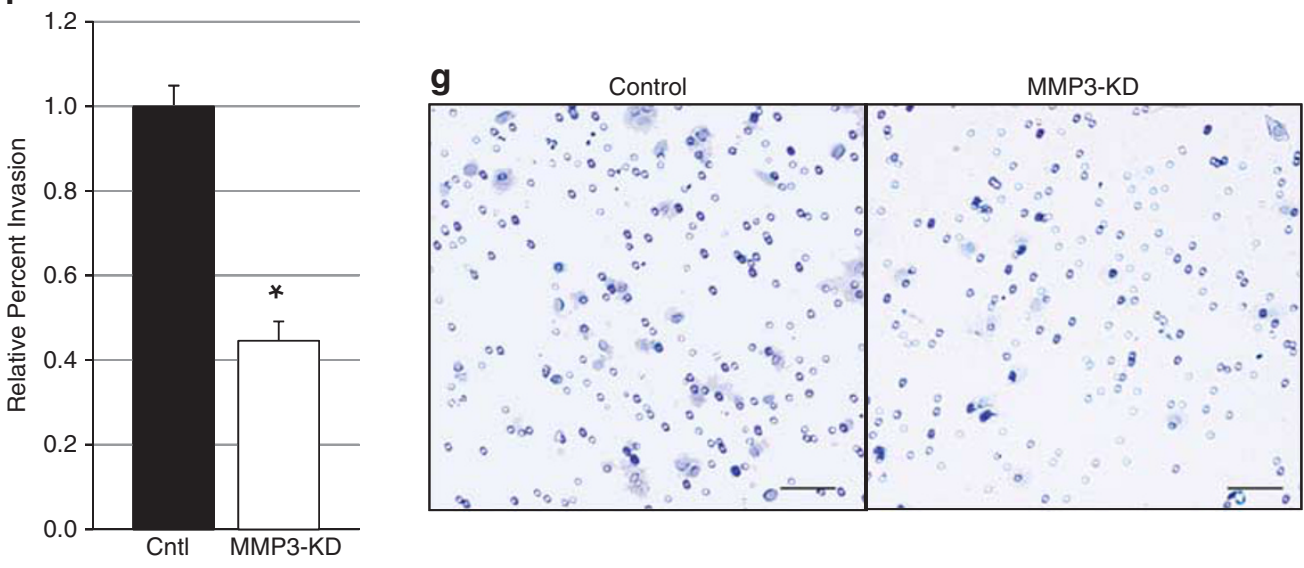

Figure 3 Expression of invasion-related genes in SOX2-KD melanoma cell line. Seven genes studied in an array of invasion-related genes had a $>80 \%$ decrease in expression in SOX2-KD cell line compared with control (a). Quantitative RT-PCR (b) and western blotting (c) of mRNA and protein expression of MMP-3 in SOX2-KD line compared with control. MMP-3 knockdown in the A2058 cell line was confirmed by quantitative RT-PCR (d) and western blotting (e). Cell invasion of MMP-3-KD cells was compared with control by in vitro invasion assay (f, $\mathbf{g})$. Data in panels $\mathbf{b}$, $\mathbf{d}$, and $\mathbf{f}$ are presented as mean \pm s.d. ${ }^{*}$ Marks significant $(P<0.05)$ differences compared with the control group.

non-infiltrative, tumorigenic vertical growth. ${ }^{22}$ Regions of tumors forming well-circumcised, coalescent nodules showed low or absent SOX2 and MMP-3 co-expression (Figures 4a and b). In contrast, more infiltrative areas showed co-positivity for SOX2 and MMP-3, particularly where nests of small cells (Figures $4 \mathrm{c}$ and $\mathrm{d}$ ) or single and spindle cell aggregates (Figures $4 \mathrm{e}$ and $\mathrm{f}$ ) invaded the adjacent stroma. This association was further examined in a tissue microarray, where SOX2/MMP-3 co-expression was consistently most intense only in cores showing stromal infiltration by melanoma cells $(n=6)$, as opposed to those composed primarily of coalescent tumor with little or no associated stroma $(n=31)$ (Figures $4 \mathrm{~g}$ and $\mathrm{h}$ ).

To further examine this preliminary observation, we analyzed subcutaneous melanoma xenografts established from A2058 SOX2-KD $(n=5)$ and control cell lines $(n=5)$ in immunodeficient mice. Correlative dual-label immunohistochemistry and immunofluorescence demonstrated preferential positivity for SOX2 and MMP-3 in tumors derived from the control line compared with SOX2KD-derived xenografts. Moreover, SOX2 and MMP-3 in tumors derived from control A2058 melanoma cells were preferentially co-expressed at tumor edges where malignant cells interfaced with and infiltrated the surrounding stroma (Figures $4 \mathrm{i}-\mathrm{p}$ ).

In summary, we found SOX2 expression by melanoma cells to be associated with an invasive behavior in vitro and with an infiltrative phenotype in situ, and have implicated MMP-3 as one potential downstream mediator of these features of melanoma virulence. 

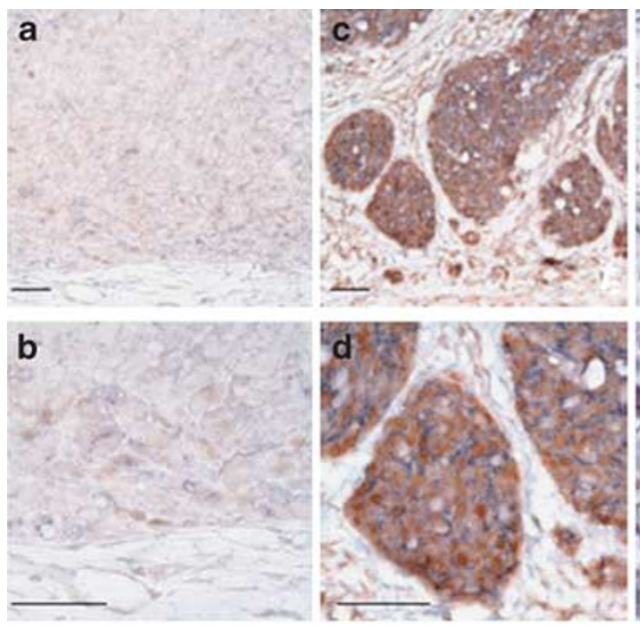

Control
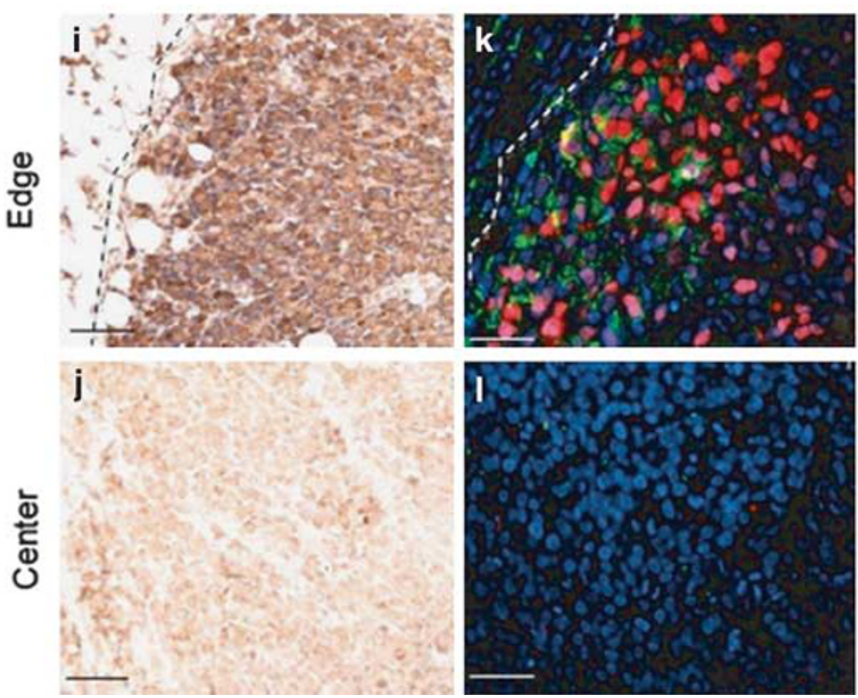
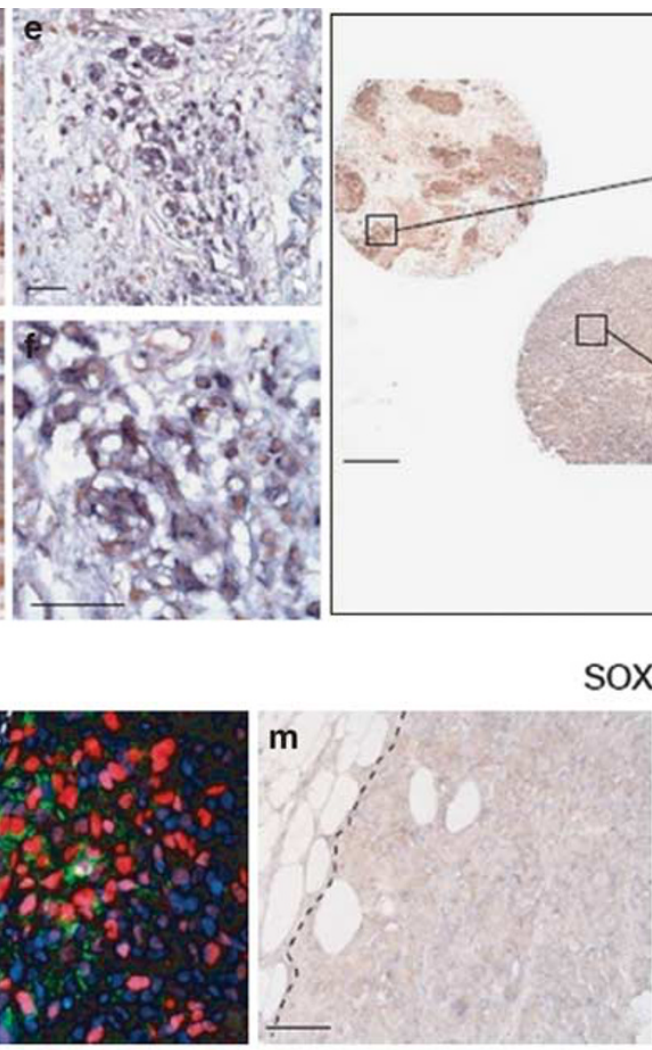

n

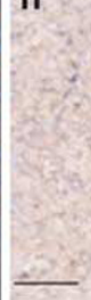

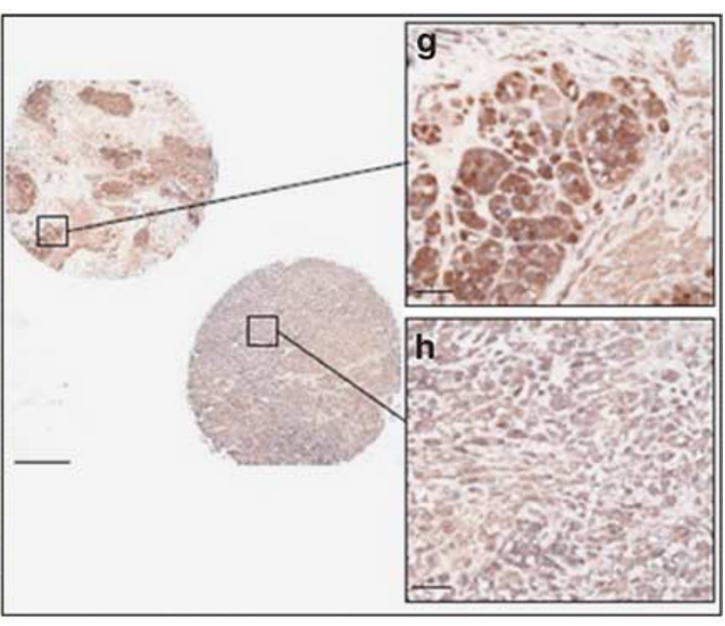

SOX2-KD
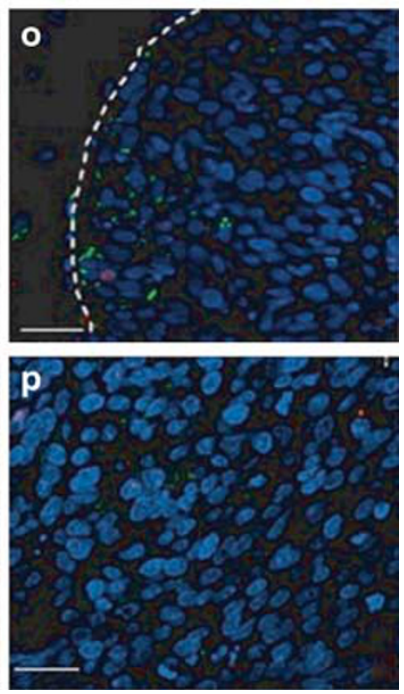

Figure 4 SOX2 and MMP-3 immunoreactivity in patient and xenograft melanomas. In patients (a-f), SOX2 (brown) and MMP-3 (blue) immunopositivity is located in small nests $(\mathbf{c}, \mathbf{d})$ or single cells $(\mathbf{e}, \mathbf{f})$ infiltrating stroma, in contrast to tumors with non-invasive perimeters (a, b). Adjacent cores from tissue microarray (TMA) (g, h) show strong SOX2/MMP-3 co-expression in small nests infiltrating stroma (g) and weaker immunostaining in expansile tumorigenic zones without stroma (h). Xenograft melanomas demonstrate preferential co-expression of SOX2 (brown by IHC, red by IF) and MMP-3

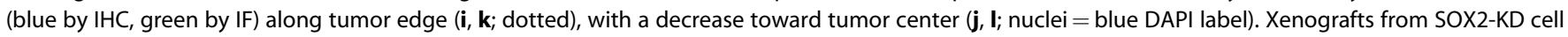
line $(\mathbf{m}-\mathbf{p})$ were relatively devoid of SOX2 and MMP-3. (a-f, i-p) Bar $=50 \mu \mathrm{m},(\mathbf{g}, \mathbf{h})$ bar $=500 \mu \mathrm{m}$.

\section{DISCUSSION}

Although the role of SOX2 in human melanoma cell invasion has not, to our knowledge, been previously studied, a relationship between SOX2 expression and invasion has been documented in other malignancies. In a recent study of human head and neck squamous cell carcinoma, spheroidderived stem-like tumor cells that expressed elevated levels of SOX2 were more invasive in Matrigel assays compared with SOX2-negative monolayer-derived cells. ${ }^{23}$ Moreover, overexpression of SOX2 in breast cancer stem cells increased their capacity for invasion in in vitro invasion assays. ${ }^{24}$ Finally, in gastric carcinoma, a statistically significant correlation was shown between SOX2 expression, depth of invasion, and patient prognosis. ${ }^{25}$
The precise mechanism whereby SOX2 expression mediates tumor cell invasion is unknown. However, it is of potential relevance that this transcription factor regulates pluripotency in embryonic neural stem cells that inherently exhibit migratory behavior. Although the factors that govern migratory characteristics of embryonic neural crest stem cells are complex, it is well recognized that ligands and receptors that mediate cell matrix interactions, motile attributes intrinsic to migratory cells, and interactions between enzymes and extracellular matrix elements have fundamental roles in this process. ${ }^{26-28}$

The association of SOX2 and MMP-3 expression noted herein raises the possibility that MMP-3 may be a downstream SOX2-regulated gene that facilitates melanoma 
invasion. MMP-3 is a member of the metalloproteinase family of extracellular matrix degrading enzymes that in malignant neoplasms facilitates tumor invasion and metastasis. ${ }^{29}$ MMP-3 cleaves a variety of substrates, including type IV collagen, laminin, and E-cadherin, a cell-cell adhesion molecule that suppresses invasion. ${ }^{29}$ Moreover, MMP-3 activates other members of the MMP family, such as MMP1 and MMP9 by cleaving their zymogen forms. ${ }^{29}$ In human melanoma, MMP-3 has been shown to be expressed in more aggressive, metastatic cell lines; facilitate melanoma cell invasion by activating latent MMP- $1 ;^{30}$ and associated with decreased disease-free survival. ${ }^{31}$ Although the association observed between SOX2 expression and MMP-3 at mRNA and protein levels in the present study raises the possibility that the latter influences the invasive behavior of SOX2positive melanoma cells, to our knowledge, a SOX2 binding site within the MMP-3 promoter sequence has not been identified and SOX2 may regulate MMP-3 gene expression indirectly.

In a preliminary analysis of patient melanomas in tissue sections and a TMA, preferential co-expression of SOX2 and MMP-3 was noted in regions of stromal infiltration by single cells or small nests of tumor cells. In addition, although staining in melanoma xenografts revealed decreased expression of both SOX2 and MMP-3 in tumors derived from the SOX2-KD line, their preferential SOX2/MMP-3 co-positivity was observed in control xenografts primarily at the tumor borders where malignant cells infiltrate surrounding stroma. This pattern for MMP-3 expression is consistent with previous reports in which staining in melanoma was confined to deeper invasive borders ${ }^{32}$ and areas adjacent to blood vessels. ${ }^{33}$ In aggregate, our findings suggest that co-expression of SOX2 and MMP-3 may serve as a functionally relevant biomarker for invasive melanoma cells.

In summary, these data extend previous observations regarding the potential role of SOX2 expression in human melanoma virulence. Melanoma invasion has clinical implications not only for primary tumors but also additionally for metastases that also require interaction with the surrounding stromal microenvironment to support growth and further spread. The mechanisms responsible for melanoma invasion, however, are undoubtedly complex, and SOX2 is likely to be one among many other contributors to the underlying pathways. Moreover, to definitively establish a role for SOX2 in melanoma invasion, additional studies are underway to examine the effects of KD and overexpression in cell lines that normally express intermediate levels of this transcription factor, as well as to better define how SOX2 manipulation affects stromal interactions within in vivo models. Further inquiries are also now indicated to explore further SOX2-associated downstream virulence factors to better understand how melanoma cells that express stem cellassociated transcriptional regulators interact with the host microenvironment.

\section{ACKNOWLEDGEMENTS}

This work was supported by the following Grants from the NIH: P50 CA93683, P30 AR042689, and R01 CA138231. We express our gratitude to Dr Jie Ma and Dr Carsten Weishaupt for help with the melanoma xenografts.

\section{DISCLOSURE/CONFLICT OF INTEREST}

The authors declare no conflict of interest.

1. Bylund $M$, Andersson $E$, Novitch $B G$, et al. Vertebrate neurogenesis is counteracted by Sox1-3 activity. Nat Neurosci 2003;6: 1162-1168.

2. Masui S, Nakatake $Y$, Toyooka $Y$, et al. Pluripotency governed by Sox2 via regulation of Oct $3 / 4$ expression in mouse embryonic stem cells. Nat Cell Biol 2007;9:625-635.

3. Kashyap V, Rezende NC, Scotland KB, et al. Regulation of stem cell pluripotency and differentiation involves a mutual regulatory circuit of the NANOG, OCT4, and SOX2 pluripotency transcription factors with polycomb repressive complexes and stem cell microRNAs. Stem Cells Dev 2009:18:1093-1108.

4. Chen Y, Shi L, Zhang L, et al. The molecular mechanism governing the oncogenic potential of SOX2 in breast cancer. J Biol Chem 2008;283:17969-17978.

5. Gure AO, Stockert E, Scanlan MJ, et al. Serological identification of embryonic neural proteins as highly immunogenic tumor antigens in small cell lung cancer. Proc Natl Acad Sci USA 2000;97:4198-4203.

6. Maier S, Wilbertz $\mathrm{T}$, Braun M, et al. SOX2 amplification is a common event in squamous cell carcinomas of different organ sites. Hum Pathol 2011;42:1078-1088.

7. Comtesse N, Zippel A, Walle $\mathrm{S}$, et al. Complex humoral immune response against a benign tumor: frequent antibody response against specific antigens as diagnostic targets. Proc Natl Acad Sci USA 2005;102:9601-9606.

8. Jia $X$, Li X, Xu Y, et al. SOX2 promotes tumorigenesis and increases the anti-apoptotic property of human prostate cancer cell. J Mol Cell Biol 2011:3:230-238.

9. Li $X L$, Eishi $Y$, Bai $Y Q$, et al. Expression of the SRY-related HMG box protein SOX2 in human gastric carcinoma. Int J Oncol 2004;24: 257-263.

10. Ben-Porath I, Thomson MW, Carey VJ, et al. An embryonic stem cell-like gene expression signature in poorly differentiated aggressive human tumors. Nat Genet 2008:40:499-507.

11. Hussenet T, Dali S, Exinger J, et al. SOX2 is an oncogene activated by recurrent 3q26.3 amplifications in human lung squamous cell carcinomas. PLoS One 2010;5:e8960.

12. Bass $\mathrm{AJ}$, Watanabe $\mathrm{H}$, Mermel $\mathrm{CH}$, et al. $\mathrm{SOX} 2$ is an amplified lineagesurvival oncogene in lung and esophageal squamous cell carcinomas. Nat Genet 2009:41:1238-1242.

13. Freier $\mathrm{K}$, Knoepfle $\mathrm{K}$, Flechtenmacher $\mathrm{C}$, et al. Recurrent copy number gain of transcription factor SOX2 and corresponding high protein expression in oral squamous cell carcinoma. Genes Chromosomes Cancer 2010;49:9-16.

14. Laga AC, Lai CY, Zhan Q, et al. Expression of the embryonic stem cell transcription factor SOX2 in human skin: relevance to melanocyte and merkel cell biology. Am J Pathol 2010;176:903-913.

15. Laga AC, Zhan $Q$, Weishaupt $C$, et al. SOX2 and nestin expression in human melanoma: an immunohistochemical and experimental study. Exp Dermatol 2011;20:339-345.

16. Schoenhals M, Kassambara A, De Vos J, et al. Embryonic stem cell markers expression in cancers. Biochem Biophys Res Commun 2009;383:157-162.

17. Thiery JP, Acloque $H$, Huang RY, et al. Epithelial-mesenchymal transitions in development and disease. Cell 2009;139:871-890.

18. Mani SA, Guo W, Liao MJ, et al. The epithelial-mesenchymal transition generates cells with properties of stem cells. Cell 2008;133:704-715.

19. Morel AP, Lievre M, Thomas $C$, et al. Generation of breast cancer stem cells through epithelial-mesenchymal transition. PLoS One 2008; 3:e2888.

20. Livak KJ, Schmittgen TD. Analysis of relative gene expression data using real-time quantitative PCR and the $2(-$ Delta Delta $C(T))$ method. Methods 2001;25:402-408. 
21. Juhasz I, Albelda SM, Elder DE, et al. Growth and invasion of human melanomas in human skin grafted to immunodeficient mice. Am J Pathol 1993;143:528-537.

22. Elder DE, Murphy GF, In: Silverberg S (ed). Melanocytic Tumors of the Skin. American Registry of Pathology: Silver Spring, MD, 2010, pp 209-276.

23. Chen C, Wei $Y$, Hummel $M$, et al. Evidence for epithelial-mesenchymal transition in cancer stem cells of head and neck squamous cel carcinoma. PLoS One 2011;6:e16466.

24. Simoes BM, Piva $M$, Iriondo $O$, et al. Effects of estrogen on the proportion of stem cells in the breast. Breast Cancer Res Treat 2011;129:23-35.

25. Matsuoka J, Yashiro M, Sakurai $K$, et al. Role of the stemness factors Sox2, Oct3/4, and Nanog in gastric carcinoma. J Surg Res 2010 (in press).

26. Tucker RP. Neural crest cells: a model for invasive behavior. Int J Biochem Cell Biol 2004;36:173-177.

27. Kalcheim C. Mechanisms of early neural crest development: from cell specification to migration. Int Rev Cytol 2000;200:143-196.
28. Frolichsthal-Schoeller $\mathrm{P}$, Vescovi AL, Krekoski CA, et al. Expression and modulation of matrix metalloproteinase-2 and tissue inhibitors of metalloproteinases in human embryonic CNS stem cells. Neuroreport 1999;10:345-351.

29. Visse $\mathrm{R}$, Nagase $H$. Matrix metalloproteinases and tissue inhibitors of metalloproteinases: structure, function, and biochemistry. Circ Res 2003;92:827-839.

30. Benbow U, Schoenermark MP, Mitchell TI, et al. A novel host/tumor cell interaction activates matrix metalloproteinase 1 and mediates invasion through type I collagen. J Biol Chem 1999;274:25371-25378.

31. Nikkola J, Vihinen $P$, Vlaykova $T$, et al. High expression levels of collagenase-1 and stromelysin-1 correlate with shorter disease-free survival in human metastatic melanoma. Int J Cancer 2002;97:432-438.

32. Walker RA, Woolley DE. Immunolocalisation studies of matrix metalloproteinases-1, -2 and -3 in human melanoma. Virchows Arch 1999;435:574-579.

33. Bodey B, Bodey Jr B, Siegel SE, et al. Matrix metalloproteinase expression in malignant melanomas: tumor-extracellular matrix interactions in invasion and metastasis. In Vivo 2001;15:57-64. 